\title{
Maguindanaon-Taw sa Laya epics Balatamay Lumëna, Diwata Kasaripan and Raha Samadaya: A reflection of their addat
}

Mangindra, Anesa $\square$

Sultan Kudarat State University, Philippines (apmangindra@gmail.com)

Sandoval, Mary Ann

Mindanao State University - Iligan Institute of Technology, Philippines (maryzann@yahoo.com)

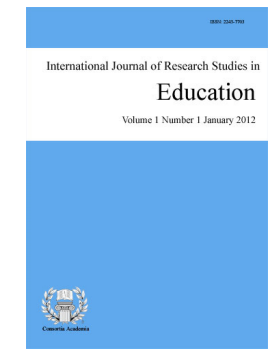

ISSN: 2243-7703 Online ISSN: 2243-7711

OPEN ACCESS

Received: 30 March 2021

Revised: 2 May 2021

Accepted: 30 May 2021

Available Online: 10 June 2021 DOI: $10.5861 /$ ijrse. 2021.626

\section{Abstract}

This study collected epics of the Maguindanaon-Taw sa Laya and translated into Filipino language. There were three epics collected, namely: Balatamay Lumëna, Diwata Kasaripan, and Raha Samadaya. Two of the epics (Diwata Kasaripan and Raha Samadaya) were personally collected by the researcher. While the Balatamay Lumëna was adopted from the collection of Dalandangan (2017). Specifically, the study aimed to discover the beliefs, customs and traditions embedded in the three epics which symbolized the addat of the Maguindanaon-Taw sa Laya. To achieve the objective, the epics were analyzed using cultural theory, new historicism, postcolonialism, acculturation, assimilation, and amalgamation. A qualitative design was utilized in the study. Descriptive-analytic and indigenous methods were also employed. The three epics were discussed through content analysis. It was found out that the three epics reflected the addat of the Maguindanaon-Taw sa Laya. Addat is derived from Maguindanaon-Taw sa Laya term which means culture' before and after the coming of Islam'. These were evident in their beliefs in supernatural beings, mystery, amulet, God, prophet, and Holy Qur'an. They were also rich in customs and traditions as the basis of their actions, behaviors, and decision makings. It implied that the addat of Maguindanaon-Taw sa Laya was a mixture of ancient and Islamic influences. The result of the study is a proof of what Alunan Glang (Madale, 1982, p. 349) has been stated, "The introduction of Islam in Maguindanao did not replace the indigenous life patterns of the people but was simply added to the pre-Islamic paganistic practices."

Keywords: addat; ancient; epic; Islam; Maguindanaon 


\section{Maguindanaon-Taw sa Laya epics Balatamay Lumëna, Diwata Kasaripan and Raha Samadaya: A reflection of their addat}

\section{Panimula}

Karaniwang maririnig sa bibig ng mga nakatatandang Maguindanaon -Taw sa Laya ang pahayag na "addat a pinaganay" na may literal na kahulugang sinaunang kultura. Batay rito, ang addat ay wikang Maguindanaon-Taw sa Laya na tumutukoy sa kultura. Subalit hindi matiyak sa nasabing pahayag kung ang tinutukoy nilang addat ay sa panahong wala pa ang Islam o hanggang sa pagdating na ng Islam sa kanilang pamayanan. Kaugnay nito, maaaring isaalang-alang bilang paglilinaw ang sinabi ni Bara (2015) tungkol sa addat na ayon sa kaniya ay kabuoan ng kultura bago pa man dumating ang mga Espanyol at sa pilosopikal na interpretasyon ng mga Muslim sa katuruan ng Islam. Ipinahihiwatig ng kaniyang pahayag na ang addat bago pa man dumating ang mga Espanyol ay maaaring ang pinagsanib na kultura bago at pagkatapos dumating ang Islam sa kapuluan.

Ang mga kabatiran tungkol sa sinaunang addat ng mga Maguindanaon-Taw sa Laya ay isang pagpapalagay lamang sapagkat hindi pa uso ang pagsulat noon. Magkagayunpaman, maaaring isaalang-alang ang sinabi ni Gowing (1988) sa pahayag niyang bago pa man ipakilala ang pananampalatayang Islam sa Mindanaw, ang buong lambak ng Pulangi o Rio Grande ay pinanahanan ng mga katutubong inugat sa lahing Malay. Ang kultura nila ay katulad din ng mga kamag-anak nilang Malay na nakatira sa patag na mga lugar sa Pilipinas na pinagtibay ng magkatulad na katangian ng kanilang sosyal at politikal na organisasyon (Dalandangan, 2017, p. 8). Ibig sabihin, ang mga tao sa buong lambak ng Pulangi na batay sa kasaysayan ay tinawag na mga Maguindanaon ay tulad din ng ibang katutubo sa Pilipinas na mayroon nang sariling kultura bago pa man dumating ang relihiyong Islam.

Maliban sa nabanggit, pinatunayan din ni McKenna (1998, pp. 47-48), ang paniniwalang mayroon nang sariling kultura ang mga Maguindanaon bago pa man dumating ang Islam sa kapuluan. Ayon sa kaniya, bago pa dumating ang Islam, ang mga Maguindanaon ay nagtataglay ng organisasyong sosyal na binubuo ng mga pangkat na may bangsa, na namumuhay sa isang partikular na inged o lokalidad. Sa Cotabato noon, ang mga pangkat na ito ay pinamumunuan ng kanilang lider na ang kapangyarihan ay hindi lamang sa sariling bangsa at inged kundi maging sa mga karatig na kaharian (sa akin ang salin). Sinusugan ito ni Stewart (1981, p. 109), na nagsabing tinatawag na timuway ang pinakamataas na pinuno ng sinaunang lipunang Maguindanaon. Nahahati sa tatlo ang kanilang katayuang sosyal gaya ng maharlika (noble), malaya (freemen) at alipin (slave). Batay sa mga nabanggit, makikitang may maayos nang sistemang panlipunan ang mga sinaunang Maguindanaon bago pa man dumating ang Islam.

Nang dumating ang Islam, lalo pang nalinang ang sistemang sosyal at politikal ng mga Maguindanaon sa pamumuno ni Sharif Kabungsuwan na nagpakilala sa kanila ng relihiyong Islam (Stewart, 1981, p. 109). Sinang-ayunan din ito ng iba pang awtor na nagsabing, "Pinasimunuan ng mga misyonerong Muslim ang pagtatag ng mga sultanato sa lipunang Maguindanaon. Ang unang naging sultan nito ay si Shariff Kabungusuwan, ang unang dumating na misyonero sa lugar. Ang dating nag-iisang sultanato ay hinati sa tatlo - Maguindanao, Buayan at Kabuntalan (Glang \& Concover, 1978, p. 21, Stewart 1981, p. 109). Sa kabila ng pagdating ng bagong pamunuan, ang kapangyarihan ng mga lokal na pinuno (timuway) ay hindi rin nawala subalit pinalitan ng terminong 'datu' ang bansag sa kanila. Ayon naman kay Shinzo (2007, p. 27), ang Maguindanao ay nalinang bilang isang ganap na kaharian pagkatapos yakapin ng mga dugong bughaw na Maguindanaon ang relihiyong Islam.

Noon pa man, di iilan ang mga dalubhasang nagpatunay tungkol sa mayamang kultura ng Maguindanaon. Kabilang dito si Gowing (1974) na nagsabing, ang mga katutubong Magindanawn lalo na ang mga nakatira sa mga liblib na pook ay naniniwala sa mga hindi kapani-paniwalang makapangyarihang nilalang tulad ng higante na si Lagasi, at si Talabusaw na ang kalahati ay tao at ang kalahati ay kabayo. Naniniwala rin sila sa busaw o asuwang. 
Naniniwala rin sila sa mantiyanak, isang uri ng asuwang na nasa pormang maliit na bata. May pagkademonyo ito kaya kinatatakutan din ng mga tao. Higit sa lahat, naniniwala sila sa tonong, ang hindi nakikitang espiritu. Ang tonong ay dalawa - ang masama na tinatawag na saytan at ang jinn na hindi masama. Ang mga hindi nakikitang espiritu ay maaaring magbigay ng sakit o kapahamakan sa mga tao. Maliban sa mga nabanggit, naniniwala rin ang mga Magindanawn sa kapangyarihan ng agimat at pusaka.

Kapag pinag-uusapan ang kulturang Maguindanaon, mapapansing tinatalakay ito sa kabuoan - hindi tiyak kung Taw sa Laya ba o Taw sa Ilud. Ngunit sa kabila ng kasalatan ng mga literatura para sa kultura ng tiyak na pangkat ng Maguindanaon, naniniwala ang mananaliksik na ang kulturang nakapaloob sa tatlong epiko ng Maguindanaon-Taw sa Laya ay tunay na nakabatay sa paraan ng pamumuhay ng nasabing pangkat sapagkat ayon kay Scott (Dalandangan, 2017, p. 53), ang katutubong panitikan bilang sining ay hindi nilikha mula sa kawalan, hindi rin simpleng likha ng isang mamamayan ang naturang panitikan bangkus ay itinuturing itong obra ng awtor na nabibilang sa isang tiyak na panahon at mahalagang kasapi ng kaniyang lipunan. Ibig sabihin, ang nilalaman ng panitikan ay nakabatay sa tiyak na panahon at lipunang ginagalawan ng mga tao. Maaari ding isaalang-alang ang pahayag ni Pagkalinawan (2006, p. 1) na nagsasabing ang panitikan ay buhay dahil ito ay batay sa pamumuhay at pakikipamuhay ng mga tao sa kaniyang ginagalawang lipunan. Ipinahihiwatig lamang nito na ang bawat akdang panitikan ng isang partikular na pangkat ay imbakan ng mga pangyayari at/o paraan ng pamumuhay sa lipunang kanilang ginagalawan.

Samantala, ang epiko bilang isa sa mga halimbawa ng katutubong panitikang patula ay punong-puno ng talinghaga, kariktan at kasiningan na palaging hinahangaan ninuman at nagiging paksa ng mga pagsusuri. Ngunit ayon kay Dalandangan (2017, p. 55), hindi sinusuri ang epiko sa pamamagitan ng kariktan at kasiningan nito, sa halip ay sinisiyasat ang naturang tulang bayan batay sa inilalarawang kultura ng nagmamay-ari nitong pangkat sapagkat ang kulturang ito ay tiyak na naglalarawan sa kanilang kasaysayan. Ibig sabihin, hindi ang panlabas na istruktura ng epiko ang dapat suriin kundi ang kulturang nakapaloob dito. Kaugnay nito, nararapat isaalang-alang ang pahayag ni Swingwood (Dalandangan, 2017) na ang salaysaying patula o epiko bilang salamin ng kasaysayan ay hindi maaaring suriin sa pamamagitan ng taglay nitong kasiningan kundi sa pagbakas at pag-analisa sa pinagmulan o pinag-ugatan, istruktura, at kulturang taglay ng nilalaman nito para sa kapakinabangan ng kasalukuyan.

Kung tutuusin, isang akdang produkto lamang ng imahinasyon ang epiko. Subalit hindi ito nangangahulugang walang pinagbatayan ang nabuo sa imahinasyon ng mga katutubong umakda nito sa pamamagitan ng kanilang dila. Sa ibang salita, ang mga salindilang akda noon ay hindi iiral kung wala itong pinagbatayang pangyayari sa pamumuhay ng ating mga ninuno. Patunay lamang na sa kabila ng pagtataglay ng epiko ng mga tagpong kababalaghan at hindi kapani-paniwala ay katatagpuan ito ng makatotohanang kultura. Pinatunayan ito ni Cruz (2003) sa pahayag niyang hindi salamin ng buhay o katotohanan ang panitikan kundi ang panitikan ay ang katotohanan mismo. Ibig sabihin, ang mga pangyayari at kulturang nakapaloob sa mga epiko ay totoong umiral noong unang panahon maging ito man ay sa panahong bago at pagkatapos dumating ang Islam.

May mangilan-ngilan na ring nagsagawa ng pag-aaral hinggil sa kulturang Maguindanaon na masasalamin sa kanilang mga katutubong panitikan tulad ng mga kuwentong bayan, epiko, awiting bayan at karunungang bayan. Kabilang sa kanila sina Dalandangan (2017), Mantikayan (2017), Panday, N. (2014), Ansa (2014), Dimaudtang (2013), Saliao (2013), Panday, A. (2008) at Caup (2003). May nakapagsagawa na rin ng pag-aaral hinggil sa epikong Magindanawn-Taw sa Laya na Balatamay Lumëna. Ang nasabing katutubong panitikan ang tinalakay ni Dalandangan (2009) kung saan tinuklas at sinuri niya ang kulturang nakapaloob dito. Ang nabanggit na mga pag-aaral ay may mga tiyak na elemento ng kulturang Maguindanaon lamang ang binigyang-tuon. Nangangahulugang hindi nasakop ang iba pang elemento ng kulturang Maguindanaon.

Samantala, maliban sa epikong Balatamay Lumëna, may ilan pang mga epiko ng Maguindanaon-Taw sa Laya ang hindi pa nagawaaan ng pag-aaral na maaaring kasasalaminan din ng makulay at mayamang kultura ng nasabing pangkat. Ang naturang kabatiran ang nagtulak sa mananaliksik upang pag-aralan ang mga epikong 
Mangindra, A., \& Sandoval, M. A.

Balatamay Lumëna, Diwata Kasaripan at Raha Samadaya kung saan tinuklas niya ang kulturang Maguindanaon-Taw sa Laya na kinategorya sa paniniwala, kaugalian, at tradisyon na may kaugnayan sa konsepto ng digmaan at kapayapaan. Mula rito, naging tunguhin ng pag-aaral na ilarawan ang addat ng Maguindanaon-Taw sa Laya. Sa pagsasakatuparan nito, naging saligan ng pagsusuri ang mga epikong Balatamay Lumëna, Diwata Kasaripan at Raha Samadaya.

\subsection{Layunin}

Pangkalahatang layunin ng pag-aaral na ito na masusing mailarawan ang addat ng Maguindanaon-Taw sa Laya na masasalamin sa kanilang katutubong panitikang epiko. Sinuri sa papel na ito ang mga diskurso mula sa mga naratibo ng mga pangyayari sa mga epikong Balatamay Lumëna, Diwata Kasaripan at Raha Samadaya.

\section{Pamamaraan}

Ginamit sa pag-aaral na ito ang disenyong kuwalitatib at kombinasyon ng pamamaraang deskriptib-analitik at indehinus. Ginamit ang pamaraang indehinus sa paglikom ng mga epiko at sa pakikipanayam at/o focused group discussion sa mga impormante at balideytor ng kulturang Maguindanaon. Ang nilalaman ng mga epikong nalikom ay tinalakay naman sa pamamagitan ng pag-aanalisa sa nilalaman. Isinagawa ang pangangalap ng mga epiko sa Lalawigan ng Maguindanao at Lalawigan ng Hilagang Cotabato. Sa Lalawigan ng Maguindanao, ang baranggay Lasangen sa bayan ng Salipada K. Pendatun ang pinagkunan ng datos samantalang ang lugar na ginamit sa Lalawigan ng Hilagang Cotabato ay ang baranggay Kabasalan sa bayan ng Pikit. Pinili ang mga impormante at balideytor sa pamamagitan ng purposive sampling upang matiyak ang kuwalipikasyon o pagtugon ng mga ito sa kraytiryang inihanda ng mananaliksik.

Tatlong (3) epiko ng mga katutubong Maguindanaon-Taw sa Laya ang ginamit sa pag-aaral bilang pangunahing datos. Sa tatlong epikong ginamit, dalawa sa mga ito ay aktuwal na kinalap ng mananaliksik. Idinaan sa transkripsiyon ang nasabing dalawang epiko. Ang impormante ng epikong Raha Samadaya ay isang literate kaya siya na rin ang gumawa ng transkripsiyon ng nasabing epiko batay sa pakiusap ng mananaliksik. Ibig sabihin, ang epikong Diwata Kasaripan lamang ang ginawaan ng transkripsiyon ng mananaliksik sapagkat hindi literate ang impormante nito. Matapos ang transkripsiyon ng dalawang epiko, isinalin ang mga ito ng mananaliksik sa wikang Filipino gamit ang meaning based translation ni Larson (1984) at pamaraang adapsiyon ni Newmark (1988). Ang mga isinaling epiko ay ipinabalideyt sa tatlong Maguindanaon-Taw sa Laya na bihasa sa larang ng Filipino. Samantala, kinuha naman mula sa disertasyon ni Dalandangan (2017) ang epikong naisalin na sa wikang Filipino. Ang nasabing epiko ay aktuwal na kinalap, isinatitik at isinalin sa wikang Filipino ni Dalandangan (2017).

Sa paghahangad ng mananaliksik na maging malinaw at mabisa ang pagtalakay sa addat ng Maguindanaon-Taw sa Laya gamit ang tatlong epiko, maingat na sinunod ang sumusunod na mga hakbang sa pagsasaayos at pag-aanalisa ng mga datos.

$>$ Una, binasa nang masinsinan ang tatlong epiko. Sa pagbabasang ito, maingat na inunawa ang addat ng Maguindanaon-Taw sa Laya batay sa mga pangyayari, kilos, pananalita, katangian, at ugali ng mga tauhan.

> Pangalawa, tinukoy ang addat mula sa tatlong epiko sa tulong ng mga pangyayari, kilos, pananalita, katangian at ugali ng mga tauhan.

$>$ Pangatlo, ipinabalideyt ang mga natukoy na kultural na paniniwala, kaugalian at tradisyon mula sa tatlong epiko upang matiyak ang katumpakan at awtentisidad ng mga mga ito. Tiglilimang balideytor na pawang Maguindanaon-Taw sa Laya ang mga balideytor na hinati sa apat na pangkat: mga matatandang nasa edad animnapu (60) pataas, mga asatidz o ulama, mga propesyonal at mga lider ng komunidad na pawang nasa edad tatlumpo't lima (35) pataas.

$>$ Pang-apat, ang mga natukoy na addat ay tinalakay at sinuri ayon sa pagkakagamit o paglitaw ng mga ito

104 Consortia Academia Publishing (A partner of Network of Professional Researchers and Educators) 
batay sa nilalalaman ng tatlong epiko na sinusuportahan ng mga literatura, pag-aaral at resulta ng pakikipanayam sa mga impormante tungkol sa kultura ng Maguindanaon-Taw sa Laya.

\section{Resulta at pagtalakay}

Inilalahad sa bahaging ito ang mga pangyayari na sumasalamin sa kultural na paniniwala, kaugalian, at tradisyon ng mga Maguindanaon -Taw sa Laya. Ginamit bilang batayan ng pagtalakay ang mga naratibo ng kanilang mga epikong Balatamay Lumëna, Diwata Kasaripan at Raha Samadaya.

\subsection{Ang Mga Paniniwala ng Maguindanaon-Taw sa Laya}

Bilang isa sa mga pangkat-etnikong Moro sa Mindanao, ang mga Maguindanaon-Taw sa Laya ay nagtataglay ng iba't ibang mga paniniwala na nagsisilbing gabay nila sa pang-araw-araw na pamumuhay. Ang nasabing mga paniniwala ay maaaring may kaugnayan sa mga di-pangkaraniwang bagay o nilalang at sa Dakilang Lumikha na nakatutulong upang lalo pang mapatatag at mapatibay ang kanilang aspetong espirituwal. Sa pamamagitan ng mga paniniwalang taglay ay nagkakaroon sila ng disiplina at kumpiyansa sa sarili na kaya nilang malampasan ang anumang dagok ng buhay. Mababasa sa unang talahanayan ang mga paniniwalang masasalamin sa tatlong epiko.

\section{Talahanayan 1}

Ang mga paniniwala ng Maguindanaon-Taw sa Laya

\begin{tabular}{|c|c|c|}
\hline Epiko & Paniniwala & Paliwanag \\
\hline Raha Samadaya & diwata at tunong & $\begin{array}{l}\text { Ang mga diwata at tunong ay nakatira sa kalikasan o sa kapaligiran. Ang mga tao ay } \\
\text { maaaring humingi ng tulong sa kanila sa oras ng pangangailangan at/o panganib. }\end{array}$ \\
\hline Balatamay Lumëna & tunong/tonong & $\begin{array}{l}\text { May dalawang uri ng tunong. Ang masamang tunong ay tinatawag na saytan } \\
\text { samantalang ang mabuti ay tinatawag na jinn. Ang saytan na maituturing na munafik } \\
\text { (masama) ay gumagawa ng mga bagay na labag sa kalooban ng Maykapal. Ang } \\
\text { nasabing tunong ay mapanganib. Ang jinn na maituturing na mabuti ay nakatutulong } \\
\text { sa mga tao sa pagpapagaling ng mga maysakit, panghuhula at paglaban sa mga } \\
\text { masasamang espiritu at tao. }\end{array}$ \\
\hline Diwata Kasaripan & Pusaka & $\begin{array}{l}\text { Ang pusaka ay tumutukoy sa lumang gamit ng mga ninunong Maguindanaon na } \\
\text { maaaring gamitin bilang panlaban sa mga sakit at proteksiyon laban sa masasamang } \\
\text { nilalang. Anumang bagay o gamit na umiral sa sinaunang lipunan ng mga } \\
\text { Maguindanaon ay itinuturing na pusaka. } \\
\text { Ang ilan sa mga pusaka ay "inigadong" (ibinaon sa lupa) at mahuhukay lamang ito } \\
\text { ng taong "ikhlas" (may busilak na kalooban). Maituturing ang pusaka na isang } \\
\text { "barakat" (mahiwaga at makapangyarihan) at ginagamit bilang sandata sa } \\
\text { pakikidigma noong unang panahon. Kinabibilangan ito ng sundang, dilek (sibat), } \\
\text { kris, k'lung, kampilan, gulok at iba pa. }\end{array}$ \\
\hline Raha Samadaya & Pusaka & $\begin{array}{l}\text { Ang pusaka ay hindi lamang nasa anyo ng mga sandata. Maituturing ding pusaka } \\
\text { ang mga sinaunang instrumentong pangmusika tulad ng agong, kulintang, } \\
\text { gandingan, debakan, kudyapi, }\end{array}$ \\
\hline Raha Samadaya & Agimat & $\begin{array}{l}\text { Bahagi rin ng kultura ng Maguindanaon-Taw sa Laya ang paniniwala sa hiwaga at } \\
\text { kapangyarihan ng agimat. May panganib man o wala ay lagi silang nagsusuot ng } \\
\text { agimat bilang proteksiyon sa kanilang sarili anumang oras na may banta ng } \\
\text { panganib sa kapaligiran na parehong dulot ng karaniwan at di -pangkaraniwang } \\
\text { nilalang. }\end{array}$ \\
\hline Balatamay Lumëna & & $\begin{array}{l}\text { May apat na anyo/uri ng agimat. Una, ang Andrun/Andlun na tumutukoy sa isang } \\
\text { maliit na lumang Qur'an buhat sa Mecca. Ikalawa, ang Datumanong na nasa anyo } \\
\text { ng magkadugtong na ulo ng ahas o tuko (ulo sa itaas at ulo rin sa ibaba/dulo). Ikatlo, } \\
\text { ang talikup na nagmula sa bahagi ng punongkahoy na "midtalikup" (nagyakapan). } \\
\text { Ikaapat, Sising Ngo Lagia Sulayman (singsing ni Raha Sulayman). Ang nasabing } \\
\text { apat na anyo o uri ng agimat ay binabalot ng telang itim at maaaring kuwintasin, } \\
\text { gawing pulseras at pinding (parang sinturon). Ang sinumang magsuot ng alinman sa } \\
\text { apat na binanggit ay hindi tatablan ng bala at anumang uri ng sandata at masasamang } \\
\text { elemento maliban na lamang kung talagang kagustuhan ni Allah (SWT). }\end{array}$ \\
\hline Diwata Kasaripan & Mahiwagang Panyo & $\begin{array}{l}\text { Sila ay naniniwala rin sa hiwaga at kapangyarihan ng panyo tulad ng kakayahan } \\
\text { nitong lumipad bilang sasakyang panghimpapawid ng mga taong may pagka-barakat } \\
\text { (natatanging kapangyarihan) noong unang panahon. Ang nasabing } \\
\text { di-pangkaraniwang kagamitan tulad ng mahiwagang panyo ay pag-aari lamang ng } \\
\text { taong may busilak na kalooban. Patunay lamang na bukod-tangi sa lipunang } \\
\text { Maguindanaon ang mga taong may busilak na kalooban habang itinatakwil nito ang } \\
\text { mga taong may maiitim na budhi. Ang pagtataglay ng mga tao ng busilak na } \\
\text { kalooban ay isa sa mga tulay upang makamit at mapanatili ang kapayapaan sa } \\
\text { lipunan. }\end{array}$ \\
\hline
\end{tabular}


Mangindra, A., \& Sandoval, M. A.

Talahanayan 1 ...pagtutuloy

\begin{tabular}{|c|c|c|}
\hline Epiko & Paniniwala & Paliwanag \\
\hline Raha Samadaya & $\begin{array}{c}\text { Busaw/Talabusaw, at } \\
\text { Lagasi }\end{array}$ & $\begin{array}{l}\text { Naniniwala rin sila na may nilalang sa mundo na kumakain ng tao. Tinatawag nila } \\
\text { itong busaw (maaaring katumbas ng aswang). Ang busaw ay maaaring nasa anyo ng } \\
\text { tao o hayop. May kakayahan ang busaw na magpalit-palit ng anyo, kaya lubha itong } \\
\text { kinatatakutan ng mga Maguindanaon-Taw sa Laya. }\end{array}$ \\
\hline Diwata Kasaripan & & $\begin{array}{l}\text { Ang mga katutubong Maguindanaon lalo na ang mga nakatira sa mga liblib na pook } \\
\text { ay naniniwala sa mga di-kapani-paniwalang makapangyarihang nilalang na sina } \\
\text { Lagasi, at Talabusaw na ang kalahati ay tao at ang kalahati ay kabayo. }\end{array}$ \\
\hline Diwata Kasaripan & $\begin{array}{l}\text { Paniniwala sa Diyos } \\
\text { at sa Kaniyang } \\
\quad \text { Propeta }\end{array}$ & $\begin{array}{l}\text { Bago pa man dumating ang Islam sa kapuluan, may sarili na silang addat. Subalit sa } \\
\text { pamamagitan ng pagdating ng Islam, unti-unting nabago ang paraan ng kanilang } \\
\text { pamumuhay batay sa katuruan ng nasabing relihiyon. }\end{array}$ \\
\hline Raha Samadaya & & $\begin{array}{l}\text { Sinalamin ng nabanggit na mga naratibo ang naging impluwensiya ng Islam sa } \\
\text { paniniwala ng mga Maguindanaon-Taw sa Laya na utang na loob nila kay Shariff } \\
\text { Kabungsuwan, ang nagpakilala ng naturang relihiyon sa Maguindanao. } \\
\text { Ang Islam, bilang relihiyon ng mga Maguindanaon, ay nangingibabaw bilang } \\
\text { pangunahing institusiyon na bumibigkis sa kanilang pangkat at nagsisilbing matibay } \\
\text { na batayan ng kanilang pakikipagkapuwa at paraan ng pagdidisiplina (Glang at } \\
\text { Convocar 1978, p. 21). Nangangahulugan lamang ito na talagang isinabuhay ng mga } \\
\text { Maguindanaon ang mga katuruang Islamiko sa kanilang pang-araw-araw na } \\
\text { pamumuhay. }\end{array}$ \\
\hline Raha Samadaya & Pagdarasal ng Subuh & $\begin{array}{l}\text { Bilang bahagi ng paniniwala ng mga Maguindanaon-Taw sa Laya sa Dakilang } \\
\text { Lumikha na nasa kalangitan, pormal silang nagdarasal ng limang beses sa isang } \\
\text { araw. Una, ang subuh na isinasagawa tuwing madaling-araw. Pangalawa, ang } \\
\text { dhuhur tuwing tanghali. Pangatlo, ang asr tuwing dapit-hapon. Pang-apat, ang } \\
\text { magrib na isinasagawa kasabay ng paglubog ng araw. Panlima, ang aisha na tuwing } \\
\text { gabi pagkatapos ng hapunan. }\end{array}$ \\
\hline Diwata Kasaripan & $\begin{array}{c}\text { Pagdarasal sa Masjid } \\
\text { (Moske) }\end{array}$ & $\begin{array}{l}\text { Kabilang din sa palatandaan ng impluwensiya ng relihiyong Islam sa kulturang } \\
\text { Maguindanaon ang pagkakaroon ng masjid (moske) sa kanilang pamayanan. Ang } \\
\text { masjid ay pook-dalanginan ng mga mananampalataya sa Islam. Dito nila karaniwang } \\
\text { isinasagawa ang salatul jum'ah (sama-samang pagdarasal) na binubuo ng dalawang } \\
\text { rak'ah o yunit na pinangungunahan ng imam. Ang salatul jum'ah ay isinasagawa } \\
\text { tuwing Biyernes ng tanghali, sa oras ng dhuhur pagkatapos ng khutbath o sermon. }\end{array}$ \\
\hline Raha Samadaya & $\begin{array}{l}\text { Pagsasagawa ng } \\
\text { Abdas Bago }\end{array}$ & $\begin{array}{l}\text { May paniniwala ang mga Maguindanaon-Taw sa Laya na kailangan munang linisin } \\
\text { ang iba't ibang bahagi ng katawan bago magsagawa ng pormal na pagdarasal. }\end{array}$ \\
\hline Diwata Kasaripan & Magsimba / Magdasal & $\begin{array}{l}\text { Tinatawag itong "abdas" sa kanilang wika, "wudu" sa Arabik at ablusyon naman sa } \\
\text { wikang Filipino. Tubig ang kanilang karaniwang ginagamit sa pag-abdas. Ang } \\
\text { pag-abdas ay tinatanaw nila hindi lamang bilang paglilinis ng mga bahagi ng } \\
\text { katawan kundi paglilinis din ng mga nagawa nilang kasalanan. Ngunit may mga } \\
\text { pagkakataong nawawalang-bisa ang kanilang abdas sanhi ng iba't ibang salik ayon } \\
\text { sa kanilang paniniwala. }\end{array}$ \\
\hline Diwata Kasaripan & $\begin{array}{c}\text { Paniniwala sa Kitab/ } \\
\text { Qur'an }\end{array}$ & $\begin{array}{l}\text { Bilang batayan ng kanilang pananampalataya, naniniwala ang mga } \\
\text { Maguindanaon-Taw sa Laya sa Kitab (Banal na Aklat). Pangunahing Kitab na } \\
\text { kanilang pinaniniwalaan ang Qur'an na naglalaman ng mga salita ni Allah (SWT), } \\
\text { ang kinikilalang Diyos ng mga Muslim. Ang Qur'an ang tinaguriang batayan ng } \\
\text { relihiyong Islam. Orihinal itong nakasulat sa wikang Arabic. }\end{array}$ \\
\hline Raha Samadaya & $\begin{array}{l}\text { Paniniwala sa } \\
\text { Kapalaran o } \\
\text { Pagtatakda ni Allah }\end{array}$ & $\begin{array}{l}\text { Naniniwala ang mga Maguindanaon-Taw sa Laya na lahat ng karanasan ng tao, } \\
\text { mabuti man o masama ay ayon sa kagustuhan ng Panginoon. Sa ibang salita, ang } \\
\text { bawat kabiguan at tagumpay na tinatamasa ng tao ay itinadhana ng Diyos at walang } \\
\text { sinuman ang makababali nito. }\end{array}$ \\
\hline
\end{tabular}

\subsection{Mga Kaugalian at Tradisyon ng Maguindanaon-Taw sa Laya}

Tulad ng iba pang etnikong pangkat na Moro, makulay at mayaman din ang mga kaugalian at tradisyon ng Maguindanaon-Taw sa Laya. Ang nasabing mga kaugalian at tradisyon ay nagsisilbing pagkakakilanlan ng kanilang pangkat. Bahagi ng pang-araw-araw nilang pamumuhay ang pagsasabuhay sa kanilang mga kaugalian at tradisyon.

Ang mga kaugalian at tradisyon ng Maguindanaon-Taw sa Laya ay masasalamin sa kanilang katutubong panitikan. Mapatutunayan ito sa mga naratibo ng mga pangyayari sa kanilang tatlong epiko - ang Balatamay Lumëna, Diwata Kasaripan at Raha Samadaya na matutunghayan sa susunod na talahanayan. 
Maguindanaon-Taw sa Laya epics Balatamay Lumëna, Diwata Kasaripan and Raha Samadaya

Talahanayan 2

Mga kaugalian at tradisyon ng Morong Magindanawn-Taw sa Laya

\begin{tabular}{|c|c|c|}
\hline Epiko & $\begin{array}{l}\text { Kaugalian at } \\
\text { Tradisyon }\end{array}$ & Paliwanag \\
\hline Diwata Kasaripan & $\begin{array}{l}\text { Pamahalaang } \\
\text { Sultanato }\end{array}$ & $\begin{array}{l}\text { Ang mga katutubong Maguindanaon ay may sarili nang sistema ng pamumuhay } \\
\text { bago pa man dumating ang Islam sa kapuluan. Pinatunayan ito ni McKenna (1998, } \\
\text { pp. 47-48), sa pahayag niyang "Bago pa dumating ang Islam, ang mga }\end{array}$ \\
\hline Raha Samadaya & & $\begin{array}{l}\text { Maguindanaon ay nagtataglay ng organisasyong sosyal na binubuo ng mga pangkat } \\
\text { na may bangsa, na namumuhay sa isang partikular na inged o lokalidad. Sa Cotabato } \\
\text { noon, ang mga pangkat na ito ay pinamumunuan ng kanilang lider na ang } \\
\text { kapangyarihan ay hindi lamang sa sariling bangsa at inged kundi maging sa mga } \\
\text { karatig na kaharian. Sinusugan ito ni Stewart (1981, p. 109), na nagsabing tinatawag } \\
\text { na timuway ang pinakamataas na pinuno ng sinaunang lipunang Maguindanaon. } \\
\text { Nahahati sa tatlo ang kanilang katayuang sosyal: maharlika (noble), malaya } \\
\text { (freemen) at alipin (slave). Makikitang may maayos ng sistemang panlipunan ang } \\
\text { mga sinaunang Maguindanaon bago pa man dumating ang Islam. } \\
\text { Masasabi ring may maayos na sistemang politikal ang mga katutubong } \\
\text { Maguindanaon-Taw sa Laya na masasaksihan sa pamumuno ng sultan sa bayan o } \\
\text { kaharian. Mahihinuha rin ang pagtataglay ng mga sultan ng mga katangiang } \\
\text { karapat-dapat angkinin ng isang pinuno, lalo na sa usapin ng pangangalaga sa } \\
\text { kaligtasan, kaayusan at kapayapaan ng bayan o kaharian. }\end{array}$ \\
\hline Raha Samadaya & Samaya & $\begin{array}{l}\text { Isa sa mga natatanging bahagi ng kulturang Maguindanaon-Taw sa Laya ang samaya } \\
\text { na nangangahulugang pangako o panata. Kailangan itong gawin o tuparin, dahil } \\
\text { kung hindi natupad ay may kaakibat na masamang pangyayari. }\end{array}$ \\
\hline Diwata Kasaripan & & $\begin{array}{l}\text { Ang samaya kaugnay ng pagkakaroon ng anak ay likas sa kultura ng mga } \\
\text { Maguindanaon-Taw sa Laya at nagkakaiba lamang sa panata o pangakong kapalit } \\
\text { kapag nakamit ang hiling. } \\
\text { Ang anumang binitiwang samaya ay kailangang tuparin upang maiwasan ang mga } \\
\text { di-kanais-nais na pangyayari. }\end{array}$ \\
\hline Diwata Kasaripan & Kambung & $\begin{array}{l}\text { Naniniwala rin sila sa kambung - ang kakayahan ng isang tao na pigilin o pawiin } \\
\text { ang ulan. } \\
\text { Sa pagsasasagawa ng kambung, may binibigkas na orasyon na tinatawag na } \\
\text { "pangalintaw" (mahiwagang kaalaman) habang nakaharap sa makapal at maitim na } \\
\text { ulap o paparating na ulan ang taong pakakambung. Maaari din itong gawin sa } \\
\text { pamamagitan ng pagguhit sa hangin ng "Sising ngo Lagia Sulayman" (singsing ni } \\
\text { Raha Sulayman) kasabay ng pagbigkas ng orasyon habang nakaharap sa maitim na } \\
\text { ulap o paparating na ulan. Ngunit may ibang paraan din ng kambung na gumagamit } \\
\text { ng walis o sandok ang taong pakakambung. Iwinawasiwas sa hangin ang nasabing } \\
\text { mga kagamitan paharap sa maitim na ulap o paparating na ulan habang bumibigkas } \\
\text { ng orasyon. } \\
\text { May nagsasabi na ang kambung ay karaniwang ginagawa ng mga sinaunang } \\
\text { Maguindanaon sa panahon ng kanilang paglalakbay kung may makapal at maitim na } \\
\text { ulap o pabagsak na ang malakas na ulan at wala silang masisilungan. Hindi nila ito } \\
\text { ginagawa tuwing umuulan na para bang pang-aliw lamang nila. Ginagawa lamang } \\
\text { nila ito kung may matibay na dahilan ang pagpawi o pagpigil ng ulan. Samantala, } \\
\text { may nagsasabi rin na ginagawa lamang ang pagkambung kapag napapansing hindi } \\
\text { pangkaraniwan ang makapal at maitim na ulap o paparating na ulan. } \\
\text { Nangangahulugan lamang ito na iba-iba ang intensiyon ng pagsasagawa ng } \\
\text { kambung. Gayunman, mahigpit nang ipinagbabawal sa kasalukuyan ang } \\
\text { pagkambung dahil sa paniniwalaang isang malaking kasalanan sa Allah (SWT) ang } \\
\text { pagpigil sa Kaniyang kagustuhan tulad ng pag-ulan. }\end{array}$ \\
\hline Diwata Kasaripan & $\begin{array}{l}\text { Pagbati ng "Assalamo } \\
\text { Alaykum" at Pagtugon }\end{array}$ & $\begin{array}{l}\text { Ang pagbabatian ng mga Maguindanaon-Taw sa Laya gamit ang wikang Arabik ay } \\
\text { karaniwan nilang sinasambit bilang pambungad kung sila'y dumarating sa mga }\end{array}$ \\
\hline Raha Samadaya & ng "Alaykumo Salam" & $\begin{array}{l}\text { kabahayan at tinutugon naman ng tao/mga taong dinatnan. Minsan, sinasambit din } \\
\text { nila ito bilang pambungad na pagbati sa nasasalubong nilang kapuwa-Muslim kahit } \\
\text { saan mang lugar. Ang naturang pagbabatian ay paraan ng paggalang at } \\
\text { pakikipagpalagayang-loob sa isa't isa na nagpapahiwatig ng kapayapaan. }\end{array}$ \\
\hline Diwata Kasaripan & Maratabat/Malatabat & $\begin{array}{l}\text { Palasak sa kulturang Maguindanaon ang pagkakaroon ng maratabat o malatabat } \\
\text { bilang kaakibat ng kanilang katayuang sosyal sa lipunan. Ibig sabihin, kapag }\end{array}$ \\
\hline Raha Samadaya & & $\begin{array}{l}\text { pinag-uusapan ang katayuang sosyal at politikal sa kulturang Maguindanaon, laging } \\
\text { nakakabit dito ang salitang maratabat na ayon kay Mastura (1988) ay binibigkas } \\
\text { ding malatabat, maatabat at/o maltabat. Sinabi ni McKenna (1998, p. 51), na ang } \\
\text { maratabat ay hango sa wikang Arabic na "martabat" na may literal na kahulugang } \\
\text { 'katayuan' (rank). Pangunahing ipinapahiwatig ng maratabat ang katayuang sosyal } \\
\text { at ikalawa ang 'karangalan' kaugnay ng katayuang sosyal." Sa ibang salita, ang taas } \\
\text { o baba ng maratabat ng pamilya o angkan ay nakadepende sa kanilang katayuang } \\
\text { sosyal. Ang mga pamilyang may matataas na katayuang sosyal tulad ng mga dugong } \\
\text { bughaw ay inaasahang mataas ang maratabat. Kaugnay nito, talagang inaalagaan } \\
\text { ang reputasyon ng pamilya o angkan. }\end{array}$ \\
\hline
\end{tabular}


Mangindra, A., \& Sandoval, M. A.

\section{Talahanayan 2}

\begin{tabular}{|c|c|c|}
\hline Epiko & $\begin{array}{c}\text { Kaugalian at } \\
\text { Tradisyon }\end{array}$ & Paliwanag \\
\hline Diwata Kasaripan & $\begin{array}{c}\text { Kapënduaya o } \\
\text { Poligamya }\end{array}$ & $\begin{array}{l}\text { Bilang Moro, ang pag-aasawa ng lalaki nang higit sa isa ay pinapayagan o } \\
\text { pinahihintulutan sa kultura ng Maguindanaon-Taw sa Laya. Tinatawag itong } \\
\text { kapenduaya sa wikang Maguindanaon at poligamya naman sa wikang Filipino. } \\
\text { Pinatunayan ito ni Stewart (1981, p. 118-119), sa pahayag niyang "Batay sa } \\
\text { pagkakaunawa ng mga Maguindanaon sa Qur'an, pinapayagan ang pag-aasawa nang } \\
\text { marami kung kayang suportahan ng lalaki ang mahigit sa isang asawa nang } \\
\text { pantay-pantay at walang kinikilingan maging sa kaniyang mga anak. Sa ibang salita, } \\
\text { ang pagpapahintulot sa poligamya ay may kondisyong nakasentro sa pagiging } \\
\text { makatarungan ng lalaki sa kaniyang mga asawa gayundin sa mga anak nito. } \\
\text { Ang pag-aasawa ng lalaki nang higit sa isa ay hindi lamang bilang pagsunod sa } \\
\text { pagpapahintulot ng Islam sa poligamya kundi isa rin itong karagdagang puntos sa } \\
\text { status symbol ng lalaki. } \\
\text { Maliban sa salik- sosyal ng pagpraktis ng kalalakihan sa poligamya, may iba pang } \\
\text { dahilan ito tulad ng pagdami ng bilang ng kababaihan. }\end{array}$ \\
\hline Diwata Kasaripan & Kabpaginasël & $\begin{array}{l}\text { Isang palasak na kaugalian ang pagturing sa pamilya bilang isang napakahalagang } \\
\text { yunit ng lipunan. Ganito rin ang pagtingin ng Maguindanaon-Taw sa Laya sa } \\
\text { pamilya. Kaugnay nito, mahalaga para sa kanila ang kabpaginasël. Ang } \\
\text { kabpaginasël ay nangangahulugang pasalitang paglalahad ng ugnayan ng } \\
\text { magkakapamilya o magkakaangkan upang magkakilala ang bawat isa. Sa ganitong } \\
\text { paraan nalalaman ang 'katupuwan' (ninuno ng angkan). } \\
\text { Ang kabpaginasël ay palasak sa kultura ng Maguindanaon-Taw sa Laya. }\end{array}$ \\
\hline Diwata Kasaripan & $\begin{array}{l}\text { Pagpapatugtog ng } \\
\text { Kulintang at Agong }\end{array}$ & $\begin{array}{l}\text { Likas sa kulturang Maguindanaon ang pagpapatugtog ng kulintang at agong. } \\
\text { Kabilang sa palatandaan ng pagiging katutubo at Moro ng mga Maguindanaon-Taw }\end{array}$ \\
\hline Balatamay Lumëna & & $\begin{array}{l}\text { sa Laya ang pagtataglay ng instrumentong pangmusika tulad ng agong at kulintang. } \\
\text { Ibig sabihin, tanging mga etnikong pangkat lamang na napapabilang sa katutubo at } \\
\text { Moro ang kakikitaan ng nasabing mga instrumentong pangmusika kung dito sa } \\
\text { Pilipinas ang pag-uusapan. } \\
\text { Ang agong at kulintang ay kasama sa pangunahing instrumentong pangmusika ng } \\
\text { Maguindanaon-Taw } \\
\text { sa Laya na napapabilang sa kanilang materyal na kultura. Palasak itong itinatanghal } \\
\text { sa mga pagdiriwang tulad ng kasal at kanduli. Maliban sa nabanggit, pinapatugtog } \\
\text { din ang agong at kulintang tuwing nagsasagawa ng rituwal na pagipat. }\end{array}$ \\
\hline Raha Samadaya & $\begin{array}{l}\text { Pagdadala ng Sandata } \\
\text { (kampilan at sundang) }\end{array}$ & $\begin{array}{l}\text { Ang mga Maguindanaon-Taw sa Laya sa sinaunang lipunan ay hindi kailanman } \\
\text { nawawalan ng sandata sa kanilang paglalakbay at pakikibaka. Ito ay bilang }\end{array}$ \\
\hline Balatamay Lumëna & $\begin{array}{l}\text { sa Paglalakbay at } \\
\text { Paggamit Nito sa } \\
\text { Digmaan }\end{array}$ & $\begin{array}{l}\text { paghahanda at proteksiyon nila sa kanilang mga sarili sa anumang nakaabang na } \\
\text { panganib at kalaban. Samakatuwid, likas sa kultura ng mga katutubong } \\
\text { Maguindanaon-Taw sa Laya ang pagtataglay ng mga sandata bilang proteksiyon sa } \\
\text { kanilang mga sarili laban sa mga masasamang nilalang. Nangangahulugan lamang } \\
\text { ito na handa silang sumabak sa digmaan sa ngalan ng katuwiran, katarungan at } \\
\text { kalayaan tungo sa pagkamit ng kapayapaan sa pamayanan o lipunan. }\end{array}$ \\
\hline
\end{tabular}

Batay sa ginawang pagsusuri at pagtalakay, natuklasang malinaw na sumasalamin sa addat ng Maguindanaon-Taw sa Laya ang mga epikong Balatamay Lumëna, Diwata Kasaripan at Raha Samadaya. Ang mga natuklasang addat ay magkahalong katutubo at impluwensiyang Islamiko.

Napapabilang sa natuklasang mga paniniwala mula sa pinagsanib na katutubo at impluwensiyang Islamiko ang mga sumusunod: paniniwala sa hiwaga at kapangyarihan ng diwata, tunong/tonong, pusaka, agimat, at mahiwagang panyo; paniniwala tungkol sa busaw/talabusaw at lagasi; paniniwala sa Diyos at sa Kaniyang Propeta; pagdarasal ng subuh; pagdarasal sa masjid; pagsasagawa ng abdas; paniniwala sa Kitab/Qur'an at paniniwala sa kapalaran o pagtatakda ng Allah (SWT).

Ang pamahalaang sultanato; pagbati ng "Assalamo Alaykum" at pagtugon ng "Alaykumo Salam"; samaya; kambung; kabpaginasël; maratabat o malatabat; kapënduaya; pagpapatugtog ng kulintang at agong at pagdadala ng sandata (kampilan/sundang) sa paglalakbay at paggamit nito sa digmaan laban sa masasamang nilalang ay napapabilang naman sa mga kaugalian at tradisyong nakapaloob sa mga epikong sinuri. Ang nasabing mga kaugalian at tradisyon ay hango rin sa pinagsanib na katutubong kultura at impluwensiyang Islamiko na kung tawagin ay addat sa wikang Maguindanaon-Taw sa Laya.

Sa pangkalahatan, ang mga natuklasang paniniwala, kaugalian, at tradisyon mula sa tatlong epiko ay magkahalong katutubo at impluwensiyang Islamiko. Ito’y larawan ng addat ng Maguindanaon-Taw sa Laya. Samakatuwid, ang Maguindanaon-Taw sa Laya bagaman yumakap sa relihiyong Islam nang panahong iyon ay hindi nila lubusang tinalikdan ang kinagisnang kultura. Hindi tuluyang nabura ng Islam ang kanilang 
Maguindanaon-Taw sa Laya epics Balatamay Lumëna, Diwata Kasaripan and Raha Samadaya

pagkakakilanlan bilang mga katutubo. Ang mga impluwensiyang Islamiko na bumuo sa kanilang pagkatao bilang Muslim ay nakadagdag lamang sa kanilang sinaunang kultura.

\section{Kongklusyon at rekomendasyon}

Tunay na hindi matatawaran ang pagtataglay ng Maguindanaon-Taw sa Laya ng makulay at mayamang addat. Pinatunayan ito ng kanilang mga paniniwala, kaugalian, at tradisyon na nakapaloob sa mga epikong Balatamay Lumëna, Diwata Kasaripan at Raha Samadaya na magkahalong katutubo at impluwensiyang Islamiko. Nangangahulugan lamang ito na ang kultura ng Maguindanaon-Taw sa Laya ay hango sa katutubo at impluwensiyang Islamiko na kumakatawan sa kanilang addat.

Ang natuklasang mga paniniwala (katutubo man o Islamiko) bilang bahagi ng addat ng Maguindanaon-Taw sa Laya ay isang patunay na nakaugat na sa kanilang kultura ang paniniwalang maaari nilang malampasan ang mga pagsubok sa buhay at mapagtagumpayan ang kanilang mga hangarin sa tulong ng mga di-pangkaraniwang nilalang o bagay at ng Dakilang Lumikha at Propeta. Kalakip din ng kanilang addat ang mga kaugalian at tradisyon na nagsisilbing gabay nila sa kanilang pagkilos, pag-uugali, pagpapasiya at pakikipagsapalaran. Ang natukoy na mga bahagi ng addat ng Maguindanaon -Taw sa Laya mula sa tatlong epiko ay salamin ng kanilang pagkatao o pagkakakilanlan mula noon magpahanggang ngayon na kapag mabatid ng iba pang pangkat-kultural ay magsisilbing tulay tungo sa pagkakaunawaan at paggalang sa isa't isa. Sa isang lipunang may pagkakaunawaan at paggalang sa kultura, kaakibat nito ang pagkakaroon ng kapayapaan.

Dahil sa pagtataglay ng mga epikong Balatamay Lumëna, Diwata Kasaripan at Raha Samadaya ng makulay at mayamang kultura ng Maguindanaon-Taw sa Laya na inirerepresenta ng kanilang addat, maaari itong gamitin bilang lunsaran ng mga aralin sa mga klase sa panitikan at kultura sa junior at senior high school. Sa kolehiyo naman, maaari itong isama sa mga talakayang pangklase sa panitikan at kultura ng mga mag-aaral na kumukuha ng BSEd medyor sa Filipino at AB Filipino. Maging sa paaralang graduwado, masterado man o doktorado sa Filipino, ay maaari ding gamitin ang salin sa Filipino ng tatlong epiko ng Maguindanaon-Taw sa Laya.

\section{Sanggunian}

Ansa, J. (2014). Larawan ng mga babae sa ilang piling kuwentong bayan ng mga Magindanawn (Tesis Pangmasteral). Kabacan, Cotabato: University of Southern Mindanao.

Bara, H. (2015). The history of the Muslims in the Philippines. Kinuha noong Oktubre 07, 2018 mula sa http://ncca.gov.ph/central-cultural-communities/the history-of-the Muslims-in-the-Philippines/

Caup, D. (2003). Values reflected in the proverbs of the three Filipino ethnic groups in Mindanao: An emic study (Dissertation). Marawi City: MSU-Main Campus.

Cruz, I. (2003). Bukud na bukod: Mga piling sanaysay. Quezon City: The University of the Philippines Press.

Dalandangan, F. (2009). Balatamay Lumëna: Epiko ng mga Magindanawn sa Balabak, Pikit, Cotabato (Master's Thesis). Davao City: Pamantasan ng Ateneo de Davao.

Dalandangan, F. (2017). Mga kwentong bayan at epikong Balatamay Lumëna: Pagmamapang pangkasaysayan sa lipunan ng mga Magindanawn Taw sa Laya (Disertasyon). Iligan City: MSU-Iligan Institute of Technology.

Dimaudtang, S. (2013). Mga kuwentong bayan ng mga Taw sa Ilud at Taw sa Laya: Pagmamapang pangkasaysayan at pangkalinangan ng mga Magindanawn (Disertasyon). Iligan City, MSU-Iligan Institute of Technology.

Glang, S., \& Concovar, M. (1978). Maguindanaon. Quezon City: UP-Diliman Press.

Gowing, P. (1988). Understaning Islam and Muslims in the Philippines. Quezon City: New Day Publisher.

Gowing, P., \& McAmis R. (1974). The Muslim Filipinos. Manila: La Solidaridad Press.

Larson, M. (1984). Meaning-based translation: A guide to cross language equivalence. USA: University Press of America, Inc.

Mantikayan, N. (2016). Klasipikasyon ng kultura sa mga kuwentong bayan ng pangkat-etnikong Maguindanaon 
Mangindra, A., \& Sandoval, M. A.

(Tesis Pangmasteral). Kabacan, Cotabato: University of Southern Mindanao.

Mastura, M. (1984). Muslim Filipino experience: A collection of essays. Quezon City: PDM Press.

McKenna, T. (1998). Muslim rulers and rebels: Everyday politics and armed separatism in the Southern

Philippines. Manila: Anvil Publishing House, Inc. https://doi.org/10.1525/9780520919648

Newmark, P. (1988). A textbook of translation. USA: Prentice Hall.

Pagkalinawan, L., Camba, M., Dela Torre R., Gonzales, E., \& Ungriano, A. (2006). Panitikan sa iba't ibang rehiyon. Valenzuela City: Mutya Publishing House, Inc.

Panday, A. (2008). Pagsusuri sa mga balyung nakapaloob sa sampung piling kuwentong bayan ng Maguindanawn: Implikasyon sa pagtuturo ng panitikan (Tesis Pangmasteral). Kabacan, Cotabato: University of Southern Minadanao.

Panday, N. (2014). Pagpapahalagang kultural sa mga awiting bayan ng tribong Magindanawn (Tesis Pangmasteral). Kabacan, Cotabato: University of Southern Minadanao.

Saliao, T. (2013). Kuwentong bayan ng Maguindanaon: Batayan sa pagbuo ng isinalarawang salaysay (Tesis Pangmasteral). Tacurong City: SKSU-ACCESS Campus.

Shinzo, H. (2007). Mindanao ethnohistory beyond nations. Maguindanao, Sangir, and Bagobo societies in East Maritime Southeast Asia. Quezon City: Ateneo de Manila University Press.

Stewart, J. (1981). History and culture of the Maguindanaon. Dansalan Quarterly Journal, 2(2). Marawi City: Dansalan College. 